\title{
Sistema plantio direto e convencional: efeito na produtividade de fibra de três cultivares de algodoeiro
}

\author{
Fernando Mendes Lamas ${ }^{1}$, Alexandre Cunha de Barcellos Ferreira ${ }^{2}$, Elio de Jesús Rodríguez \\ de La Torre ${ }^{3}$, Luiz Alberto Staut ${ }^{1}$
}

${ }^{1}$ Empresa Brasileira de Pesquisa Agropecuária, Centro de Pesquisa Agropecuária do Oeste, Dourados, Mato Grosso do Sul, Brasil. E-mail: fernando.lamas@embrapa.br, staut@terra.com.br

${ }^{2}$ Empresa Brasileira de Pesquisa Agropecuária - Embrapa Arroz e Feijão, Santo Antônio de Goiás, Goiás, Brasil. Email: alexandre-cunha.ferreira@embrapa.br

${ }^{3}$ Instituto Mato-grossense de Algodão, Primavera do Leste,. Mato Grosso, Brasil. E-mail: eliodelatorre@gmail.com

Recebido: 09/01/2016; Aceito: 16/05/2016.

\section{RESUMO}

O sistema de produção predominante na cultura do algodoeiro é o sistema convencional de manejo do solo, que tem proporcionado degradação dos atributos físicos, químicos e biológicos do solo. Com o objetivo de avaliar o efeito do Sistema Plantio Direto (SPD) e do Sistema Convencional de Manejo do Solo (SC) sobre a produtividade de fibra das cultivares BRS Cedro, BRS Jatobá e BRS Araçá, foram conduzidos experimentos em Primavera do Leste, MT, no período de 2006/2007 a 2012/2013. O algodoeiro foi cultivado em SPD, obedecendo à seguinte sequência para os períodos de verão e outono-inverno, respectivamente: ano 1) soja/Urochloa ruziziensis; ano 2) milho + U. ruziziensis/U. ruziziensis; ano 3) algodão/pousio; ano 4) soja/U. ruziziensis e em SC, com mobilização do solo e sem rotação de culturas. O delineamento experimental utilizado foi o de blocos ao acaso, com quatro repetições, com parcelas subdivididas: nas parcelas $(10 \mathrm{~m}$ x $30 \mathrm{~m})$ foram alocados os sistemas de manejo de solo e nas subparcelas $(10 \mathrm{~m} \times 10 \mathrm{~m})$ as cultivares. $\mathrm{O}$ sistema de manejo do solo, não tem efeito sobre a produtividade de fibra.

Palavras-chave: Gossypium hirsutum; plantio na palha; sistema de produção.

\section{No tillage and conventional management systems: effects on fiber yield of three cotton cultivars}

\section{ABSTRACT}

The predominant production system on the cotton crop is the conventional system of soil management, which has provided physical, chemical and biological degradation of the soil. The effect of no tillage (NT) and conventional system (CS) on fiber yield of BRS Cedro, BRS Jatobá and BRS Araçá were investigated in this study. The experiments were conducted in Primavera do Leste, MT in the period from 2006/2007 to 2012/2013. The cotton crop was grown in NT, in the following sequence for the periods of summer and autumn-winter, respectively: $1^{\text {st }}$ year) soybean / Urochloa ruziziensis; $2^{\text {nd }}$ year) Maize $+U$. ruziziensis $/ U$. ruziziensis; $3^{\text {th }}$ year) Cotton $/$ fallow; $4^{\text {th }}$ year) Soybean / U. ruziziensis and CS with soil management and without crop rotation. The experimental design was randomized block design with four replications, with split plots: the plots $(10 \mathrm{~m}$ x $30 \mathrm{~m})$ were allocated the land management systems and the subplots $(10 \mathrm{~m}$ x $10 \mathrm{~m})$ cultivars. The soil management system, has no effect on the fiber productivity.

Key words: Gossypium hirsutum; planting in the straw; production system. 


\section{Introdução}

A cultura do algodoeiro, no estado de Mato Grosso, nas últimas safras, atingiu níveis de produtividade equivalentes aos melhores do mundo. No ano agrícola 2014/2015 foram cultivados, nesse estado, 562.700 ha com algodoeiro, obtendo-se a produtividade de fibra de 1.638 ha $^{-1}$. Neste ano, a produção de fibra em Mato Grosso correspondeu a $61,58 \%$ da produção brasileira (CONAB, 2015).

No que se refere aos sistemas de manejo do solo utilizados, para o algodoeiro cultivado no período de safra (semeadura realizada em dezembro), predomina o Sistema Convencional (SC), com a utilização de aração + gradagens de discos "plantio semidireto" sobre palha de milheto, onde o número de gradagens é variável, mas nunca inferior a duas. Estes sistemas de manejo podem levar à degradação das propriedades físicas, químicas e biológicas do solo e, paulatinamente, diminuir o seu potencial produtivo (SEGUY; BOUZINAC, 2012).

Mais recentemente, um novo modelo de produção surge com o algodoeiro sendo cultivado após a colheita da soja. Neste modelo, a semeadura do algodoeiro é realizada sobre restos culturais da soja, que, além de pequena quantidade, apresenta características que favorecem a sua rápida decomposição. Muitas vezes, a semeadura é realizada em solo com elevado teor de umidade, o que não é recomendado. Além disso, geralmente não se faz a rotação de culturas (SALTON; LAMAS, 2015).

A degradação do solo tem sido uma preocupação constante da comunidade científica por conta da redução da produtividade das culturas, do aumento nos custos de produção e dos danos ao ambiente. Uma das alternativas, mais efetivas e eficientes de conservação do solo é a utilização do Sistema Plantio Direto (SPD) (FAGERIA; STONE, 2004).

No entanto, para a implantação e condução do SPD de maneira sustentável além do não revolvimento do solo é imprescindível a rotação de culturas a fim de manter permanentemente uma quantidade mínima de palha na superfície do solo (CRUSCIOL et al., 2010).

Avaliando diversos sistemas de manejo, em solos de textura média e muito argilosa, Carvalho et al. (2004) e Varner et al. (2011) concluíram que a produtividade de algodão não difere quando o SPD é comparado com outros sistemas de manejo.

De acordo com Corrêa e Sharma (2004), o uso do SPD pode diminuir o índice de infestação de plantas daninhas e, desta forma, reduzir o custo de produção da cultura do algodoeiro. Com adequado aporte de palha, o controle de plantas daninhas pode ser superior a $90 \%$ (MATEUS et al., 2004; FERREIRA et al.). O acúmulo de biomassa pelas plantas daninhas é inversamente proporcional ao da biomassa das plantas de cobertura (MESCHEDE et al., 2007). Por outro lado, a quantidade de palha aportada anualmente no sistema pode interferir nos processos de mineralização e imobilização do nitrogênio (ACOSTA et al., 2014).

A qualidade do solo representa o fator primordial da sustentabilidade agrícola. Entre as principais variáveis utilizadas para avaliá-la, destacam-se atributos relacionados com a dinâmica da matéria orgânica e a diversidade dos microrganismos. Tem sido observado que os teores de carbono orgânico encontrados estáveis sob vegetação natural apresentam redução acentuada com o preparo do solo, sendo afetados especialmente pela intensidade de revolvimento e pela cobertura da superfície do solo (MOORE et al., 2000). A utilização de métodos convencionais de preparo tende a reduzir os teores de carbono orgânico, resultante do aumento da taxa de decomposição anual ou redução da taxa de adição de material orgânico (DALAL; MAYER, 1986a, b). Por outro lado, o SPD pode promover aumento considerável nos teores de carbono orgânico e de nutrientes, principalmente nas camadas mais superficiais do solo (ALVAREZ et al., 1995).

O SPD, além de seus efeitos sobre o solo, afeta o crescimento das plantas em função da alteração do ambiente. Lopes et al. (2009) constataram o estabelecimento mais rápido de um índice de área foliar maior em plantas de milho cultivadas em sistema "convencional", o que repercutiu positivamente sobre o acúmulo de fitomassa total, o número de espigas e a produtividade de grãos.

O SPD requer cuidados na sua implantação e, depois de estabelecido, seus benefícios estendem-se não apenas ao solo, mas, consequentemente, ao rendimento das culturas e à competitividade dos sistemas agropecuários. Além disso, em decorrência da drástica redução da erosão, reduz o potencial de contaminação do ambiente e dá ao agricultor maior garantia de renda. Desta forma, a estabilidade da produção é ampliada em comparação aos métodos tradicionais de manejo de solo (CRUZ et al., 2001).

Além das vantagens anteriormente comentadas, quando se analisa a eficiência energética do SPD em relação ao SC, constata-se eficiência energética da ordem de $52,72 \%$ do custo do sistema SC, o que proporciona economia de óleo diesel de 24,45 L para cada hectare trabalhado (FERNANDES et al., 2008).

O objetivo do trabalho foi avaliar o efeito do sistema plantio direto e do sistema convencional de manejo do solo sobre a produtividade de fibra de três cultivares do algodoeiro.

\section{Material e Métodos}

Os experimentos foram conduzidos entre os anos agrícolas de 2006/2007 e 2012/2013, na área experimental do Instituto Mato-grossense do Algodão 
(IMAmt), em Primavera do Leste-MT, cujas coordenadas geográficas são: $15^{\circ} 31^{\prime} 53.9^{\prime \prime} \mathrm{S}$ $54^{\circ} 12^{\prime} 09.5^{\prime \prime} \mathrm{W}$, com altitude de $627 \mathrm{~m}$. O solo da área experimental é um Latossolo Vermelho-Amarelo distrófico (Embrapa, 2006), com as características químicas, na profundidade de $0-20 \mathrm{~cm}$, quando do início dos experimentos: são: $\mathrm{pH}($ Água $)=6,10 ; \mathrm{pH}\left(\mathrm{CaCl}_{2}\right)=$ 5,$45 ; \mathrm{Al}^{+++}=0,0 \mathrm{cmol}_{\mathrm{c}} \mathrm{dm}^{-3} ; \mathrm{Ca}^{++}=2,47 \mathrm{cmol}_{\mathrm{c}} \mathrm{dm}^{-3}$, $\mathrm{Mg}^{++}=0,91^{\prime} \mathrm{cmol}_{\mathrm{c}} \mathrm{dm}^{-3}, \mathrm{H}+\mathrm{Al}=2,60 \mathrm{cmol}_{\mathrm{c}} \mathrm{dm}^{-3}, \mathrm{~K}^{+}=$ $0,21 \mathrm{cmol}_{\mathrm{c}} \mathrm{dm}^{-3}, \mathrm{P}=19,21 \mathrm{mg} \mathrm{dm}^{-3}$, soma de bases $=$ $3,56 \mathrm{cmol}_{\mathrm{c}} \mathrm{dm}^{-3}, \mathrm{CTC}=6,17 \mathrm{cmol}_{\mathrm{c}} \mathrm{dm}^{-3}$, CTCef $=3,56$ $\mathrm{cmol}_{\mathrm{c}} \mathrm{dm}^{-3}, \mathrm{~V}=56,35 \%$ de saturação de bases e $19,40 \mathrm{~g}$ $\mathrm{kg}^{-1}$ de matéria orgânica. $\mathrm{O}$ solo da área experimental apresenta $595 \mathrm{~g} \mathrm{~kg}^{-1}$ de areia, $39 \mathrm{~g} \mathrm{~kg}^{-1}$ de silte e $366 \mathrm{~g}$ $\mathrm{kg}^{-1}$ de argila. $\mathrm{O}$ delineamento experimental utilizado foi o de blocos ao acaso, em parcelas subdivididas, com quatro repetições. Nas parcelas de $300 \mathrm{~m}^{2}$ (10 m x 30 m) foram alocados os tratamentos (Tabela 1), que corresponderam aos sistemas de manejo: a) - SC sistema convencional de preparo do solo (aração + gradagem pesada + gradagens niveladoras (2), com monocultivo do algodoeiro e b) SPD - sistema plantio direto, com a seguinte sequência de culturas no verão: algodão-soja-milho. Após a colheita das parcelas cultivadas com soja foi realizada a semeadura de Urochloa ruziziensis. Nestas parcelas, após a dessecação da $U$. ruziziensis com glifosato na dose de $1.400 \mathrm{~g} \mathrm{ha}^{-1}$, utilizando-se $2001 \mathrm{ha}^{-1}$ de calda, realizouse, no mês de outubro de cada ano, a semeadura do milho. Nas parcelas cultivadas com milho, quando da segunda adubação em cobertura, nas entrelinhas, foi realizada a semeadura de $U$. ruziziensis, que foi dessecada 30 dias antes da semeadura do algodoeiro.

Para eliminar o efeito de ano, em todas as safras, nos dois sistemas de manejo, foi cultivado algodoeiro. As cultivares de algodoeiro BRS Cedro - porte alto ciclo longo, BRS Jatobá - porte médio e ciclo médio e BRS Araçá - porte baixo e ciclo curto, foram alocadas nas subparcelas (10 m x $10 \mathrm{~m})$. A semeadura do algodoeiro, em todos os anos, foi realizada na primeira quinzena do mês de dezembro e a colheita entre os meses de junho e julho. Utilizou-se o espaçamento de $0,90 \mathrm{~m}$ entre fileiras com 8 a 10 plantas $\mathrm{m}^{-1}$. A adubação utilizada foi de $300 \mathrm{~kg} \mathrm{ha}^{-1}$ do adubo formulado 5-30-15, durante o período em que os experimentos foram conduzidos.

A primeira adubação em cobertura foi realizada quando do surgimento dos primeiros botões florais, com $50 \mathrm{~kg}$ de $\mathrm{N} \mathrm{ha}{ }^{-1}$, e a segunda por ocasião do aparecimento das primeiras flores, com $50 \mathrm{~kg}$ de $\mathrm{N} \mathrm{ha}^{-1}$ mais $60 \mathrm{~kg}$ de $\mathrm{K}_{2} \mathrm{O} \mathrm{ha}{ }^{-1}$. Como fonte de $\mathrm{N}$ utilizou-se o sulfato de amônio e como fonte de $\mathrm{K}_{2} \mathrm{O}$ o cloreto de potássio. Para o controle de plantas daninhas, pragas e doenças foram utilizados os herbicidas, inseticidas e fungicidas indicados para uso na cultura do algodoeiro, quando necessário.

As semeaduras da soja e do milho foram realizadas na segunda quinzena do mês de novembro de cada ano, utilizando-se para soja o espaçamento entre fileira de $0.45 \mathrm{~m}$ com 12 a 14 plantas por m e para o milho 0,90 $\mathrm{m}$ entre fileira com seis plantas por $\mathrm{m}$. As práticas culturais para a soja e para o milho foram aquelas preconizadas para a região.

Durante a condução dos experimentos, os principais insetos-pragas foram: pulgão - Aphis gossypii (Hemiptera: Aphidae); curuquerê - Alabama argilácea (Lepidoptera: Noctuidae); lagarta-das-maçãs (Heliothis virescens e Helicoverpa zea (Lepidopetera: Noctuidae), Bicudo: Anthonomus grandis (Coleoptera: Curculioidae) que foram controlados observando-se os fundamentos do Manejo Integrado de Pragas e os produtos recomendados por (SANTOS, 2015).

As principais espécies de plantas daninhas presentes na cultura do algodoeiro, independente do sistema de manejo de solo foram: picão preto - Bidens spp; apagafogo - Althernanthera tenella; carrapicho rasteiro Acanthospermun australe; capim-carrapicho - Cenchrus echinatus; trapoeraba - Commelina benghalensi; capimpé-de-galinha - Eleusina indica. O manejo das plantas daninhas foi realizado utilizando herbicidas seletivos para o algodoeiro.

$\mathrm{Na}$ Figura 1 está representada graficamente a precipitação pluviométrica ocorrida na área experimental nos anos agrícolas de 2008/2009 a 2012/2013.

Tabela 1. Sequência de culturas utilizadas nos sistemas de manejo estudados.

\begin{tabular}{cccccccc}
\hline Sistema & $2006 / 2007$ & $2007 / 2008$ & $2008 / 2009$ & $2009 / 2010$ & $2010 / 2011$ & $2011 / 2012$ & $2012 / 2013$ \\
\hline SCSR & Algodão & Algodão & Algodão & Algodão & Algodão & Algodão & Algodão \\
SPD1 & So/U.ruz & M+U.ruz & Algodão & So/U.ruz & M+U.ruz & Algodão & So/U.ruz \\
SPD2 & M+U.ruz & Algodão & So+U.ruz & M+U.ruz & Algodão & So/U.ruz & M+U.ruz \\
SPD3 & Algodão & So/U.ruz & M+U.ruz & Algodão & S/ U. ruz & M+ U.ruz & Algodão \\
\hline
\end{tabular}

SCSR = sistema convencional sem rotação de culturas; SPD = Sistema Plantio Direto; So/U. ruz = soja e Urochloa ruziziensis; M+U. ruz. $=$ Milho $+U$. ruziziensis . 


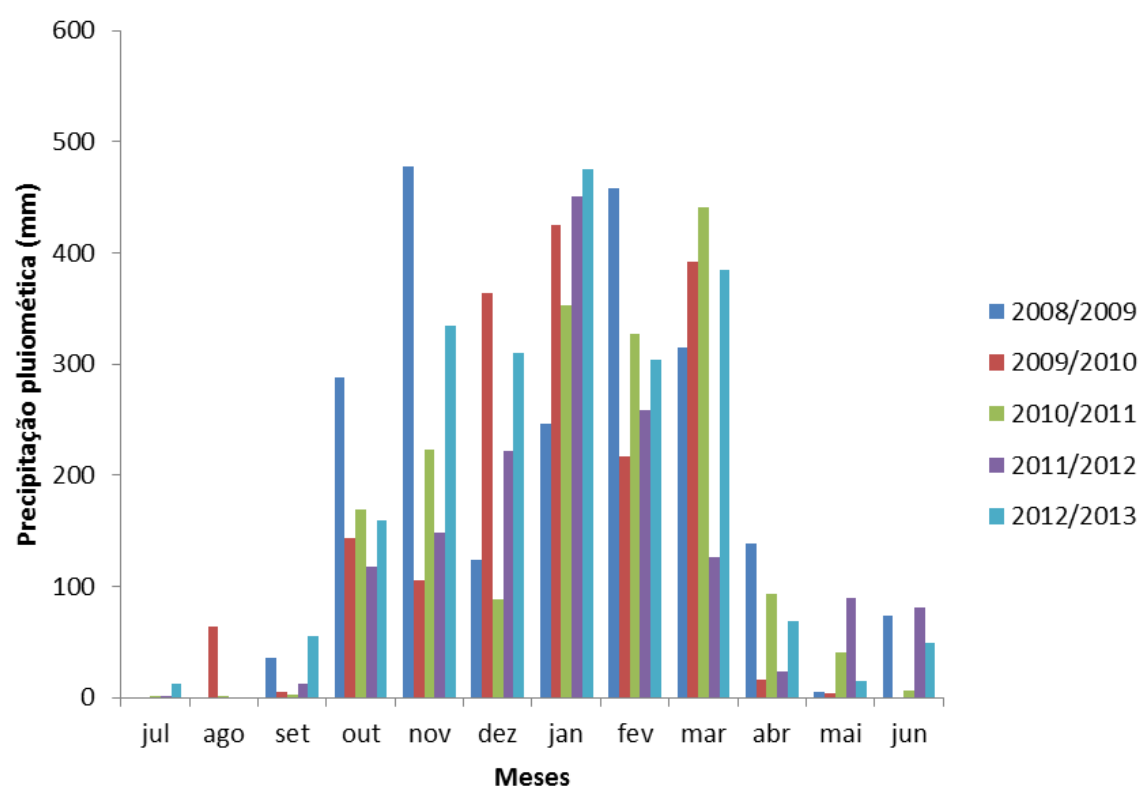

Figura 1. Precipitação pluviométrica $(\mathrm{mm})$ ocorrida na área experimental em Primavera do Leste-MT, durante os anos agrícolas de 2008/2009 a 2012/2013.

A produtividade de algodão em caroço foi determinada colhendo-se as duas linhas centrais, com 9 $\mathrm{m}$ de comprimento, de cada unidade experimental, perfazendo área de $16,2 \mathrm{~m}^{2}$. A produção obtida em cada unidade experimental, após o descaroçamento em máquina de rolo e a determinação da percentagem de fibra foi convertida em quilogramas por hectare. Os dados obtidos foram submetidos à análise de variância conjunta, considerando o ano como parcela, o sistema de manejo e as cultivares como subparcelas. As análises estatísticas foram realizadas utilizando o programa Estat. As médias dos tratamentos alocados nas parcelas foram comparadas pelo teste $\mathrm{t}$ e as demais pelo teste de Tukey, a 5\% de significância (BANZATTO; KRONKA, 1992).

\section{Resultados e Discussão}

$\mathrm{O}$ efeito dos fatores ano, sistema de manejo, cultivar e as interações ano x sistema de manejo e ano x cultivar, para a produtividade de fibra, foi significativo ( $\mathrm{P}<$ 0,05) (Tabela 2).

No período de cultivo, verificou-se diferença significativa $(\mathrm{P}<0,05)$ entre os sistemas de manejo apenas em 2008/2009 e 2010/2011, onde a média de produtividade do SC foi superior à do SPD (Tabela 3). Observou-se também menor variação da produtividade de fibra, entre os anos, no SPD.

Em análise de solos de amostras coletadas após a colheita do experimento conduzido em 2010/2011, detectou-se a necessidade de correção dos teores de potássio, que no SPD eram inferiores aos do SC. O menor teor de potássio no SPD, especialmente na camada de 10-20 cm (0,06 cmolc dm-3), pode explicar a diferença de produtividade entre os sistemas. Em amostras coletadas em 2013, ficou evidenciado que o teor de matéria orgânica do solo (MOS), na camada de 0 $-5 \mathrm{~cm}$ no SPD foi significativamente maior que no SC. O mesmo verificou-se para densidade do solo, porosidade total, macroporosidade e microporosidade A partir da camada de $5-10 \mathrm{~cm}$, não foi detectada diferença significativa entre os tratamentos. Guareschi et al. (2012), obtiveram resultados semelhantes. A magnitude das alterações dos teores de MOS sob sistemas de plantio direto ou convencional, é altamente dependente da interação de fatores edafoclimáticos, do tempo de implantação do experimento, onde quão maior o tempo de implantação, mais significativos são benefícios do SPD.

Nos dois sistemas de manejo estudados, a produtividade de fibra variou entre os anos (Tabela 3). Meredith Junior et al. (2012), ao estudarem os efeitos do ambiente, do genótipo e da interação entre esses sobre um conjunto de variáveis quantitativas e qualitativas do algodoeiro, concluíram que a produtividade de fibra é altamente influenciada pelo ambiente, o que explica a variabilidade entre os anos. O efeito do sistema de cultivo e da rotação de culturas sob a estabilidade do rendimento das diferentes espécies cultivadas depende fundamentalmente de gestão (SINDELAR et al., 2015). Em trabalhos desenvolvidos por Debiasi et al. (2013), constatou-se efeito positivo do SPD sobre a produtividade de soja, em anos com restrição hídrica na fase reprodutiva. No presente trabalho, não se constatou restrições hídricas significativas, especialmente na fase de frutificação (Figura 1). 
De acordo com Corrêa e Sharma (2004) e Ferreira et al. (2010), a cultura anterior ao algodoeiro, em SPD, pôde interferir significativamente na produtividade de fibra. De acordo com Echer et al. (2012), quando cultivado em área anteriormente ocupada com $U$. ruziziensis, o algodoeiro tem o seu crescimento reduzido pela imobilização do nitrogênio causada pelas raízes de U. ruziziensis. Em trabalho desenvolvido por Toliver et al. (2012), os autores concluem que elementos do clima (precipitação pluviométrica) e do solo (textura) podem impactar o rendimento relativo do algodoeiro quando cultivado no SPD. Boquet et al. (2004), em estudo realizado durante sete anos sobre o efeito do sistema de manejo de solo, de plantas de cobertura e doses de nitrogênio, concluíram que a produtividade de fibra de algodão era função das plantas de cobertura e da dose de nitrogênio. Na maioria das vezes, a dose de nitrogênio necessária para maximizar a produção em SPD é maior que a do SC. Na Tabela 4 são apresentados os resultados de produtividade de fibra $\left(\mathrm{kg} \mathrm{ha}{ }^{-1}\right)$, desmembrando o efeito de ano em função das cultivares.

Tabela 2. Resumo da análise de variância para produtividade de fibra.

\begin{tabular}{lcc}
\hline F.V. & Quadrados Médios & F \\
\hline Ano(A) & 3451597,12 & $63,58^{* *}$ \\
\hline Resíduo(a) & $\mathbf{5 4 2 8 5 , 0 5}$ & $3,51^{\text {ns }}$ \\
\hline Sistema de Manejo (SM) & 120126,14 & $6,61^{* *}$ \\
A x SM & 226479,69 & $4,34^{*}$ \\
\hline Resíduo (b) & $\mathbf{3 4 2 4 5 , 6 3}$ & $2,52^{* *}$ \\
\hline Cultivar (C) & 134103,79 & $1,03^{\text {ns }}$ \\
A x C & 77853,03 & $0,86^{\text {ns }}$ \\
SM x C & 31990,01 & \\
A x SM X C & 27521,41 & \\
\hline Resíduo (c) & $\mathbf{3 0 8 6 8 , 2 1}$ & \\
\hline CV (a) & 14,40 & 11,43 \\
CV (b) & 10,86 & \\
CV (c) & & \\
\hline$* *, *{ }^{\text {ns }}-$ significativo a 1\%, a 5\% e não significativo, respectivamente, pelo teste F. & \\
\hline
\end{tabular}

Tabela 3. Produtividade de fibra $\left(\mathrm{kg} \mathrm{ha}^{-1}\right)$ em função do ano e dos sistemas de manejo convencional sem rotação de culturas (SCSR) e plantio direto (SPD).

\begin{tabular}{lcc}
\hline Ano & \multicolumn{2}{c}{ Sistema de manejo } \\
\cline { 2 - 3 } & SCSR & SPD \\
\hline $2006 / 2007$ & $2.039 \mathrm{aA}$ & $1.952 \mathrm{aA}$ \\
$2007 / 2008$ & $2.006 \mathrm{aA}$ & $2.069 \mathrm{aA}$ \\
$2008 / 2009$ & $2.001 \mathrm{aA}$ & $1.594 \mathrm{bcB}$ \\
$2009 / 2010$ & $1.499 \mathrm{bcA}$ & $1.623 \mathrm{bA}$ \\
$2010 / 2011$ & $1.081 \mathrm{dA}$ & $879 \mathrm{~dB}$ \\
$2011 / 2012$ & $1.244 \mathrm{cdA}$ & $1.356 \mathrm{cA}$ \\
$2012 / 2013$ & $1.638 \mathrm{bA}$ & $1662 \mathrm{bA}$ \\
\hline Média & 1.644 & 1.591 \\
\hline
\end{tabular}

Médias seguidas pela mesma letra minúscula, na coluna, e pela mesma letra maiúscula, na linha, não diferem entre si pelos testes de Tukey e t, a 5\%, respectivamente.

Tabela 4. Produtividade de fibra $\left(\mathrm{kg} \mathrm{ha}^{-1}\right)$ em função do ano e da cultivar.

\begin{tabular}{lccc}
\hline Ano & \multicolumn{3}{c}{ Cultivares } \\
\cline { 2 - 4 } & BRS Cedro & BRS Jatobá & BRS Araçá \\
\hline $2006 / 2007$ & $2.061 \mathrm{aA}$ & $1.879 \mathrm{abA}$ & $2.045 \mathrm{aA}$ \\
$2007 / 2008$ & $2.047 \mathrm{aAB}$ & $2.151 \mathrm{aA}$ & $1.916 \mathrm{abB}$ \\
$2008 / 2009$ & $1.848 \mathrm{abA}$ & $1.741 \mathrm{bcA}$ & $1.804 \mathrm{abcA}$ \\
$2009 / 2010$ & $1.600 \mathrm{bcA}$ & $1.545 \mathrm{cA}$ & $1.539 \mathrm{cdA}$ \\
$2010 / 2011$ & $1.149 \mathrm{dA}$ & $995 \mathrm{dAB}$ & $797 \mathrm{eB}$ \\
$2011 / 2012$ & $1.415 \mathrm{cdA}$ & $1.241 \mathrm{dA}$ & $1.246 \mathrm{dA}$ \\
$2012 / 2013$ & $1.597 \mathrm{bcA}$ & $1.613 \mathrm{bcA}$ & $1.741 \mathrm{bcA}$ \\
\hline Média & 1.674 & 1.595 & 1.584 \\
\hline
\end{tabular}

Médias seguidas pela mesma letra minúscula, na coluna, e pela mesma letra maiúscula, na linha, não diferem entre si pelo teste de Tukey a 5\%, respectivamente. 
Fixando-se a cultivar e variando o ano, verifica-se diferença $(\mathrm{P}<0,05)$ entre os anos para todas as cultivares e dentro de cada ano, observando-se forte efeito do fator ano. Quando se analisa o comportamento de cada cultivar em função do ano, constata-se que no ano de 2007/2008 a produtividade de fibra da cultivar BRS Jatobá não diferiu significativamente da BRS Cedro e diferiu da BRS Araçá; no ano 2010/2011 a produtividade de fibra da cultivar BRS Cedro diferiu significativamente $(\mathrm{P}<0,05)$, apenas da cultivar BRS Araçá. Nos demais anos analisados, não se verificou diferença significativa entre as cultivares $(\mathrm{P}>0,05)$ (Tabela 3). Portanto, o efeito de ano é mais evidente do que o da cultivar, o que está em acordo com Meredith Junior et al. (2012) que, ao avaliarem o efeito do ambiente, da cultivar e a interação entre estes, constataram que o efeito do ambiente é muito mais marcante para a maioria dos parâmetros qualitativos e quantitativos avaliados. Debiasi et al. (2013), comparando o efeito do sistema de manejo de solo sobre a produtividade de soja, verificaram que, nos primeiros anos do experimento, a produtividade no SC era superior à do SPD. Somente após o sexto ano verificouse superioridade do SPD. Os mesmos autores afirmam que este período pode ser superior, dependendo da quantidade de palha que anualmente é aportada ao sistema.

\section{Conclusões}

1. Para produtividade de fibra, o efeito da cultivar independe do sistema de manejo de solo.

2. O algodoeiro pode ser um dos componentes do sistema de produção em SPD.

\section{Referências Bibliográficas}

CONAB. COMPHANIA NACIONAL DE ABASTECIMENTO. Décimo primeiro levantamento da safra brasileira grãos. Brasília-DF: Conab, 2015. Disponível em: http://www.conab.gov.br/OlalaCMS/uploads/arquivos/15_11_ 16_15_18_26_safras_nov_2015.pdf. Acesso em: 29 nov. 2015

ACOSTA, J. A. de A.; AMADOR, T. J. C.; SILVA, L. S. da; SANTI, A.; Weber, M. A. Decomposição da fitomassa de plantas de cobertura e liberação de nitrogênio em função da quantidade de resíduos aportada ao solo sob sistema plantio direto. Ciência Rural, Santa Maria-RS, v. 44, n. 5, p. 801809, 2014.

ALVAREZ, R.; DÍAZ, R. A.; BARBERO, N.; SANTANATOGLIA, O. J.; BLOTTA, L. Soil organic carbon, microbial biomass and $\mathrm{CO}_{2}-\mathrm{C}$ production from three tillage systems. Soil and Tillage Research, Oxford, v. 33, n. 1, p. 17-28, 1995.
BANZATTO, D. A.; KRONKA, S. N. Experimentação Agrícola. Jaboticabal-SP: FUNEP, 1992. 247 p.

BOQUET, D. J.; HUTCHINSON, R. L.; BREITENBECK, G. A. Long-term tillage, cover crop, and nitrogen rate effects on cotton. Agronomy Journal, Madison, v. 96, n. 5, p. 14431452, 2004.

CARVALHO, M. A. C. de; ATHAYDE, M. L. F.; SORATTO, R. P.; ALVES, M. C.; SÁ, M. E. de. Adubação verde e sistemas de manejo do solo na produtividade do algodoeiro. Pesquisa Agropecuária Brasileira, Brasília-DF, v. 39, n. 12, p. 1205-1211, 2004.

CORRÊA, J. C.; SHARMA, R. D. Produtividade do algodoeiro herbáceo em plantio direto no Cerrado com rotação de culturas. Pesquisa Agropecuária Brasileira, Brasília-DF, v. 39, n. 1, p. 41-43, 2004.

CRUSCIOL, C. A. C.; SORATTO, R. P.; BORGHI, E.; MATEUS, G. P. Benefits of integrating crops and tropical pastures as system of production. Better Crops International, Atlanta, v. 94, n. 1, p. 14-16, 2010.

CRUZ, J. C.; PEREIRA FILHO, I. A.; ALVARENGA, R. C.; SANTANA, D. P. Plantio direto e sustentabilidade do sistema agrícola. Informe Agropecuário, Belo Horizonte-MG, v. 22, n. 208 , p. $13-24,2001$.

DALAL, R. C.; MAYER, R. J. Long-term trends in fertility of soils under continuous cultivation and cereal cropping in Southern Queesland. I. Overall changes in soil properties and trends in winter cereal yields. Australian Journal of Soil Research, Melbourne, v. 24, n. 2, p. 265-279, 1986a.

DALAL, R. C.; MAYER, R. J. Long-term trends in fertility of soils under continuous cultivation and cereal cropping in Southern Queesland. II. Total organic and its rate of loss from the soil profile. Australian Journal of Soil Research, Melbourne, v. 24, n. 2, p. 281-292, 1986 b.

DEBIASI, H.; FRANCHINI, J. C.; CONTE, O.; BALBINOT JUNIOR, A. A.; TORRES, E.; SARAIVA, O. F.; OLIVEIRA, M. C. N. de. Sistemas de preparo do solo: trinta anos de pesquisas na Embrapa Soja. Londrina-PR: Embrapa Soja, 2013. 72 p. (Documentos, 342).

ECHER, F. R.; CASTRO, G. S. A.; BOGIANI, J. C.; ROSOLEM, C. A. Crescimento inicial e absorção de nutrientes pelo algodoeiro cultivado sobre palhada de Brachiaria ruziziensis. Planta Daninha, Viçosa-MG, v. 30, n. 4, p. 783-790, 2012.

EMBRAPA. EMPRESA BRASILEIRA DE PESQUISA AGROPECUÁRIA. Sistema brasileiro de classificação de solos. 2. ed. Rio de Janeiro-RJ: Embrapa-SPI, 2006. 286 p.

FAGERIA, N. K.; STONE, C. F. Produtividade de feijão no Sistema Plantio Direto com aplicação de zinco. Pesquisa Agropecuária Brasileira, Brasília-DF, v. 39, n. 1, p. 73-78, 2004.

FERNANDES, H. C.; SILVEIRA, J. C. M. da; RINALDI, P. C. N. Avaliação do custo energético de diferentes operações agrícolas mecanizadas. Ciência \& Agrotecnologia, LavrasMG, v. 32, n. 5, p. 1582-1587, 2008. 
FERREIRA, A. C. B.; LAMAS, F. M.; CARVALHO, M. da C. S.; SALTON, J. C.; SUASSUNA, N. D. Produção de biomassa por cultivos de cobertura do solo e produtividade do algodoeiro em plantio direto. Pesquisa Agropecuária Brasileira, Brasília-DF, v. 45, n. 6, p. 546-553, 2010.

FERREIRA, A. C. B; LAMAS, F. M. Espécies vegetais para cobertura do solo: influência sobre plantas daninhas e a produtividade do algodoeiro em sistema plantio direto. Ceres, Viçosa-MG, v. 57, n. 6, p. 778-786, 2010.

GUARESCHI, R.F.; PEREIRA, M.G.; PERIN, A. Deposição de resíduos vegetais, matéria orgânica leve, estoques de carbono e nitrogênio e fósforo remanescente sob diferentes sistemas de manejo no cerrado goiano. Revista Brasileira de Ciência do Solo, Vicosa-MG, v. 36, n.3, p. 909-920, 2012.

LOPES, J. P.; MACHADO, E. C.; DEUBER, R.; MACHADO, R. S. Análise de crescimento e trocas gasosas na cultura de milho em plantio direto e convencional. Bragantia, Campinas-SP, v. 68, n. 4, p. 839-849, 2009.

MATEUS, G. P.; CRUSCIOL, C. A. C.; NEGRISLOI, E. Palhada de sorgo guiné gigante no estabelecimento de plantas daninhas em áreas de plantio direto. Pesquisa Agropecuária Brasileira, Brasília-DF, v. 39, n. 6, p. 539-542, 2004.

MEREDITH JUNIOR, W. R.; BOYKIN, D. L.; BOURLAND, F. M.; CALDWELL, W. D.; CAMPBELL, B. T.; GANNAWAY, J. R.; GLASS, K.; JONES, A. P.; MAY, L. M.; SMITH, C. W.; ZHANG, J. Genotype X environment interactions over seven years for yield, yield components, fiber quality and gossypol traits in the regional high quality tests. Journal of Cotton Science, Bossier City, v. 16, n. 1, p. 60-169, 2012.

MESCHEDE, D. K.; FERREIRA, A. B.; RIBEIRO JÚNIOR, C. C. Avaliação de diferentes coberturas na supressão de plantas daninhas no Cerrado. Planta Daninha, Viçosa-MG, v. 25, n. 3, p. 465-471, 2007.
MOORE, J. M.; KLOSE, S.; TABATABAI, M. A. Soil microbial biomass carbon and nitrogen as affected by cropping systems. Biology and Fertility of Soils, Switzerland,,v. 31,v.3-4, p. 200-210, 2000.

SALTON, J. C.; LAMAS, F. M. Cultivo do algodoeiro no sistema de integração lavoura-pecuária e o cultivo do algodoeiro nos cerrados. In: FREIRE, E.C. (Ed.). Algodão no cerrado do Brasil. Brasília-DF: ABRAPA, 2015. p. 241-265.

SANTOS, W. J. Manejo das pragas do algodão, com destaque para o cerrado brasileiro. In: FREIRE, E.C. (Ed.). Algodão no cerrado do Brasil. Brasília-DF: ABRAPA, 2015. p. 267-364.

SEGUY, L.; BOUZINAC, S. Alternativas de plantio direto de alta performance. In: Belot, J.-L. (Ed.). Manual de boas práticas de manejo do algodoeiro em Mato Grosso. CuiabáMT: IMAmt, 2012. p. 38-43.

SINDELAR, A. J.; SCHMER, M. R.; JIN, V. L.; WIENHOLD, B. J.; VARVEL, G. E. Long-term corn and soybean response to crop rotation and tillage. Agronomy Journal, Madison, v. 107, n. 6, p. 2241- 2252, 2015.

TOLIVER, D. K.; LARSON, J. A.; ROBERTS, R. K.; ENGLISH, B. C.; UGARTE, D. G. De La T.; WEST, T. O. Effects of no-till on yields as influenced by crop and environmental factors. Agronomy Journal, Madison, v. 104, n. 2, p. 530-541, 2012.

VARNER, B. T.; EPPLIN, F. M.; STRICKLAND, G. L. Economics of no-till versus tilled dryland cotton, grain sorghum, and wheat. Agronomy Journal, Madison, v. 103, n. 5, p. 1329-1338, 2011. 